\begin{tabular}{|c|c|}
\hline Title & Optimum transmit beamforming in an interactive wireless relay system with network coding \\
\hline Author(s) & Matsuya, Sakiko; Webber, Julian; Nishimura, Toshihiko; Ohgane, Takeo; Ogawa, Y asutaka \\
\hline Citation & $\begin{array}{l}\text { 2010 IEEE 21st International Symposium on Personal Indoor and Mobile Radio Communications (PIMRC), 2093-2098 } \\
\text { https://doi.org/10.1109PIMRC.2010.5671651 }\end{array}$ \\
\hline Issue Date & $2010-09$ \\
\hline DOC URL & http:/hdl.handle.net/2115/49039 \\
\hline Rights & $\begin{array}{l}\text { C 2010 IEEE. Reprinted, with permission, from Sakiko Matsuya, Julian Webber, Toshihiko Nishimura, Takee Ohgane, } \\
\text { and Y asutaka Ogawa, Optimum Transmit Beamforming in an InteractiveW ireless Relay System with Network Coding, } \\
2010 \text { IEEE } 21 \text { st International Symposium on Personal Indoor and Mobile Radio Communications (PIMRC), Sep. } 2010 \text {. } \\
\text { This material is posted here with permission of the IEEE. Such permission of the IEEE does not in any way imply IEEE } \\
\text { endorsement of any of Hokkaido University products or services Internal or personal use of this material is permitted. } \\
\text { However, permission to reprint//republish this material for advertising or promotional purposes or for creating new } \\
\text { collective works for resale or redistribution must be obtained from the IEEE by writing to pubs permissions@ ieee.org. } \\
\text { By choosing to view this document, you agree to all provisions of the copyright laws protecting it. }\end{array}$ \\
\hline Type & proceedings (author version) \\
\hline File Information & 2010PIMRC_2093-2098.pdf \\
\hline
\end{tabular}

Instructions for use 


\title{
Optimum Transmit Beamforming in an Interactive Wireless Relay System with Network Coding
}

\author{
Sakiko Matsuya, Julian Webber, Toshihiko Nishimura, Takeo Ohgane, and Yasutaka Ogawa \\ Graduate School of Information Science and Technology \\ Hokkaido University \\ Sapporo 060-0814, Japan \\ Email: \{matsuyas, julian.webber, nishim, ohgane, ogawa\}@m-icl.ist.hokudai.ac.jp
}

\begin{abstract}
Wireless relay is an essential technology to achieve a flexible network for ubiquitous communication. To improve throughput performance in the network, network coding (NC) has been proposed for a two-user relay node. $\mathrm{NC}$ is a concept encoding both users' data into one packet and multicasting it simultaneously. However, if the relay node has two or more antenna elements, antenna pattern control is additionally required to send the packet efficiently to both users. In this paper, we formulate several transmit-beamforming techniques in the interactive wireless relay system and compare the performance with each other and with non-NC systems. Numerical analysis has shown that the method maximizing the total capacity in NC provides the best performance.
\end{abstract}

\section{INTRODUCTION}

Recently, wireless meshed network, one of the realization procedures of the ubiquitous environment, has attracted much attention. Since this system builds networks without communication infrastructures, it is possible to communicate at places where a base station cannot be located.

In these wireless meshed networks, each terminal becomes not only an end user but also a relay node switching relay packets. In such a case, network coding (NC) [1] is known as one of the techniques to improve network throughput. The NC that encodes two user's data into one packet using exclusive OR (XOR) at the relay node can reduce the number of transmission packets efficiently and simply. And, physicallayer NC (PHY-NC) has also been proposed as one of the modifications [2] [3].

When encoded packets at the two-user relay node are multicasted to both users, we have to keep the required quality of the received signals at both ends. When the relay node has one antenna element, there are no degrees of freedom except adaptive modulation and coding. However, when the relay node has two or more antenna elements, control of the beam pattern is possible. Such a technique has been considered only for PHY-NC [4].

In XOR-based NC (XOR-NC), the required condition for beamforming is simple, i.e., to satisfy directing beams to both users. It is very easy to form a beam when the users are located closely, whereas two degrees of freedom are needed to form two different beams when the spatial signatures of users are not the same. If we choose to form two peaks in the beam pattern, directivity gain would be decreased due to antenna resource consumption. In contrast, one narrow beam makes it possible to maximize the gain of either user. Thus, it can be said that the optimum beamforming should be determined with a trade off between each user's signal quality. Unfortunately, such a strategy has never been discussed.

On the other hand, systems not using NC are particularly suitable for a relay system with multiple antennas since only unicast is executed. Actually, PHY-NC also fits a beamforming system since it transmits the different data simultaneously as in [2] [3]. Therefore, the performance advantage of XOR-NC might be reduced when relay beamforming is considered.

In the paper, we consider several criteria to determine the optimum transmit beamforming and formulate the optimum weight vectors for each case of XOR-NC. As a comparison, beamforming for other systems not using XOR-NC is also summarized. The rest of the paper is organized as follows. First, the system model used in the paper is defined in Section II. The details on the transmit beamforming technique are described in Sections III and IV. In Section V, the performance is numerically evaluated. Finally, we conclude the paper in Section VI.

\section{SySTEM MODEL}

As shown in Fig. 1, we consider that users $\mathrm{A}$ and $\mathrm{B}$ interactively transmit packets via a relay node $\mathrm{R}$ where the relay node transmits packets to both users at the same time slot with or without NC. In this paper we assume that users A and B have a single antenna and that the relay node $\mathrm{R}$ has $N$ $(>1)$ antenna elements. In actual situations, the users $A$ and B possibly receive the desired packet sent to the relay from another user. Then, diversity gain can be obtained by packet combining between the direct and relayed packets [5]. For the sake of simplicity, however, only the packet sent via a relay is considered here.

Let the bit-sequences of packets from the users $\mathrm{A}$ and $\mathrm{B}$ be represented as $\mathbf{S}_{\mathrm{A}}$ and $\mathbf{S}_{\mathrm{B}}$, respectively. In $\mathrm{NC}$, XOR of $\mathbf{S}_{\mathrm{A}}$ and $\mathbf{S}_{\mathrm{B}}\left(\mathbf{S}_{\mathrm{A}} \oplus \mathbf{S}_{\mathrm{B}}\right)$ is calculated at the relay node $\mathrm{R}$, and then it is transmitted to the users $A$ and $B$. To decode $\mathbf{S}_{B}$ from the received packet data $\mathbf{S}_{\mathrm{A}} \oplus \mathbf{S}_{\mathrm{B}}$ at the user A, we may simply apply XOR operation with $\mathbf{S}_{\mathrm{A}}$ known perfectly. Thus, the number of time-slots necessary for the packet transmission to two users from the relay node $\mathrm{R}$ is one.

In the case without $\mathrm{XOR}-\mathrm{NC}$, it is necessary to transmit the different packets carrying $\mathbf{S}_{\mathrm{A}}$ and $\mathbf{S}_{\mathrm{B}}$ to each user. In 


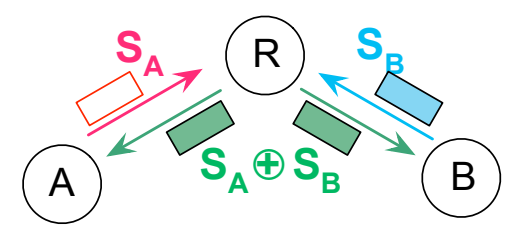

(a) NC-system

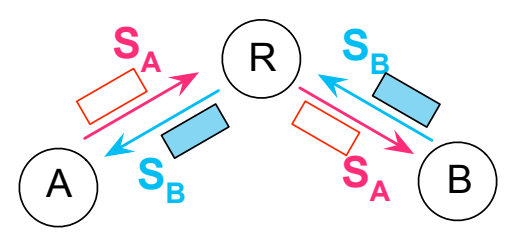

(b) non-NC-system

Fig. 1. A wireless relay system where A and B are end users, and R is a relay.

general, it requires unicast twice. Thus, two time-slots are necessary. Meanwhile, using array pattern control of the transmit antennas, two packets can be sent simultaneously with two different beams. Such a system can be implemented by PHY-NC and space division multiple access (SDMA) [6]. The former cancels inter-user interference (IUI) at the receiver side, whereas the latter suppresses the IUI by null directing. Here, SDMA and PHY-NC are also evaluated for comparison to XOR-NC.

In the following, we formulate the transmit beamforming technique for XOR-NC and others. Hereinafter, the $N$ dimensional channel vector of users $\mathrm{A}$ and $\mathrm{B}$ is denoted as $\boldsymbol{h}_{a}$ and $\boldsymbol{h}_{b}$, respectively.

\section{BEAMFORMING FOR XOR-NC}

In XOR-NC, it is necessary to transmit the packet encoded at the relay node $\mathrm{R}$ to users $\mathrm{A}$ and $\mathrm{B}$ with high quality. In this section, we formulate the following four criteria. Here, the $N$ dimensional transmit-weight vectors are commonly denoted by $\boldsymbol{w}$ for the sake of convenience.

\section{A. Omni-directional Pattern}

The simplest technique is to transmit the packet for all users with the same quality in the average sense. This can be done by an omni-directional antenna. In this paper, only the first antenna is used for signal transmission. Thus, all signal power is concentrated to one element. The weight vector $\boldsymbol{w}$ is expressed as

$$
\boldsymbol{w}_{\mathrm{o}}=[1,0, \ldots, 0]^{T} .
$$

\section{B. Maximizing SNR}

This criterion maximizes the sum of SNR at both receivers. The sum is written as

$$
P=\left(\left|\boldsymbol{h}_{a}^{T} \boldsymbol{w}\right|^{2}+\left|\boldsymbol{h}_{b}^{T} \boldsymbol{w}\right|^{2}\right) \frac{P_{S}}{P_{N}},
$$

where $P_{S}$ and $P_{N}$ are the transmitted signal power and noise power at the receiver, respectively. Maximizing $P$ given by (2) is equivalent to maximizing

$$
\left|\boldsymbol{h}_{a}^{T} \boldsymbol{w}\right|^{2}+\left|\boldsymbol{h}_{b}^{T} \boldsymbol{w}\right|^{2} .
$$

Here, we use the method of Lagrange multipliers under the constraint $\boldsymbol{w}^{H} \boldsymbol{w}=1$. The cost function can be written as

$$
J=\left|\boldsymbol{h}_{a}^{T} \boldsymbol{w}\right|^{2}+\left|\boldsymbol{h}_{b}^{T} \boldsymbol{w}\right|^{2}-\mu\left(\boldsymbol{w}^{H} \boldsymbol{w}-1\right),
$$

where $\mu$ is a Lagrange multiplier. From $\partial J / \partial \boldsymbol{w}=0$, we have

$$
\left(\boldsymbol{h}_{a}^{*} \boldsymbol{h}_{a}^{T}+\boldsymbol{h}_{b}^{*} \boldsymbol{h}_{b}^{T}\right) \boldsymbol{w}=\mu \boldsymbol{w} .
$$

Thus, the optimal transmit weight vector $\boldsymbol{w}_{\mathrm{s}}$ is the eigenvector corresponding to the maximum eigenvalue of $\boldsymbol{h}_{a}^{*} \boldsymbol{h}_{a}^{T}+\boldsymbol{h}_{b}^{*} \boldsymbol{h}_{b}^{T}$.

Clearly, $\boldsymbol{w}_{\mathrm{s}}$ is a vector in the column space of $\boldsymbol{h}_{a}^{*} \boldsymbol{h}_{a}^{T}+\boldsymbol{h}_{b}^{*} \boldsymbol{h}_{b}^{T}$, i.e., $\boldsymbol{w}$ is expressed by a linear combination of $\boldsymbol{h}_{a}^{*}$ and $\boldsymbol{h}_{b}^{*}$. If $\boldsymbol{w}$ includes the vector component orthogonal to $\boldsymbol{h}_{a}^{*}$ and $\boldsymbol{h}_{b}^{*}$, the corresponding transmitted signal does not reach the receiver. Therefore, any transmit weight $\boldsymbol{w}$ must be given as linear combination of $\boldsymbol{h}_{a}^{*}$ and $\boldsymbol{h}_{b}^{*}$ to minimize the transmit power loss.

Equation (3) implies that the total SNR maximization might emphasize an imbalance of user SNR, since improving the high SNR user would often achieve a higher total SNR. In other words, the low SNR user's performance degrades more. In such a case, it is predicted that packet error rate might become worse than that of other systems.

\section{Maximizing Capacity}

This criterion maximizes the sum of the channel capacity of both users. The sum is expressed as

$$
\begin{aligned}
C & =\log \left(1+\frac{P_{S}}{P_{N}}\left|\boldsymbol{h}_{a}^{T} \boldsymbol{w}\right|^{2}\right)+\log \left(1+\frac{P_{S}}{P_{N}}\left|\boldsymbol{h}_{b}^{T} \boldsymbol{w}\right|^{2}\right) \\
& =\log \left(1+\frac{P_{S}}{P_{N}}\left|\boldsymbol{h}_{a}^{T} \boldsymbol{w}\right|^{2}\right)\left(1+\frac{P_{S}}{P_{N}}\left|\boldsymbol{h}_{b}^{T} \boldsymbol{w}\right|^{2}\right) .
\end{aligned}
$$

Here, let us denote $\boldsymbol{w}$ by a linear combination of $\boldsymbol{h}_{a}^{*}$ and $\boldsymbol{h}_{b}^{*}$ as described above as

$$
\boldsymbol{w}=a \frac{\boldsymbol{h}_{a}^{*}}{\sqrt{A}}+b \frac{\boldsymbol{h}_{b}^{*}}{\sqrt{B}},
$$

where $a$ and $b$ are complex coefficients and $A=\boldsymbol{h}_{a}^{H} \boldsymbol{h}_{a}, B=$ $\boldsymbol{h}_{b}^{H} \boldsymbol{h}_{b}$.

From $\left|\boldsymbol{h}_{a}^{H} \boldsymbol{h}_{b}\right|^{2} \leq A B$, we define $\boldsymbol{h}_{a}^{H} \boldsymbol{h}_{b}=\sqrt{A B} \rho e^{j \phi}$ where $\rho$ and $\phi$ are constants for $0 \leq \rho \leq 1$ and $0 \leq \phi<2 \pi$. Then, we have

$$
\begin{aligned}
\left|\boldsymbol{h}_{a}^{T} \boldsymbol{w}\right|^{2} & =A\left(|a|^{2}+\rho^{2}|b|^{2}+2 \operatorname{Re}\left\{a b^{*} \rho e^{j \phi}\right\}\right) \\
\left|\boldsymbol{h}_{b}^{T} \boldsymbol{w}\right|^{2} & =B\left(|b|^{2}+\rho^{2}|a|^{2}+2 \operatorname{Re}\left\{a b^{*} \rho e^{j \phi}\right\}\right) .
\end{aligned}
$$

From (8) and (9), we can see that the phases of $a$ and $b$ affect $\left|\boldsymbol{h}_{a}^{T} \boldsymbol{w}\right|^{2}$ and $\left|\boldsymbol{h}_{b}^{T} \boldsymbol{w}\right|^{2}$ commonly. Thus, the condition for the phases of $a$ and $b$ maximizing (8) and (9) is solved as such that $a b^{*} \rho e^{j \phi}$ has a positive real value, i.e., $a b^{*}=|a||b| e^{-j \phi}$.

Unfortunately, it is difficult to draw an analytical result on the optimum amplitude of $a$ and $b$ maximizing (6). Therefore, 


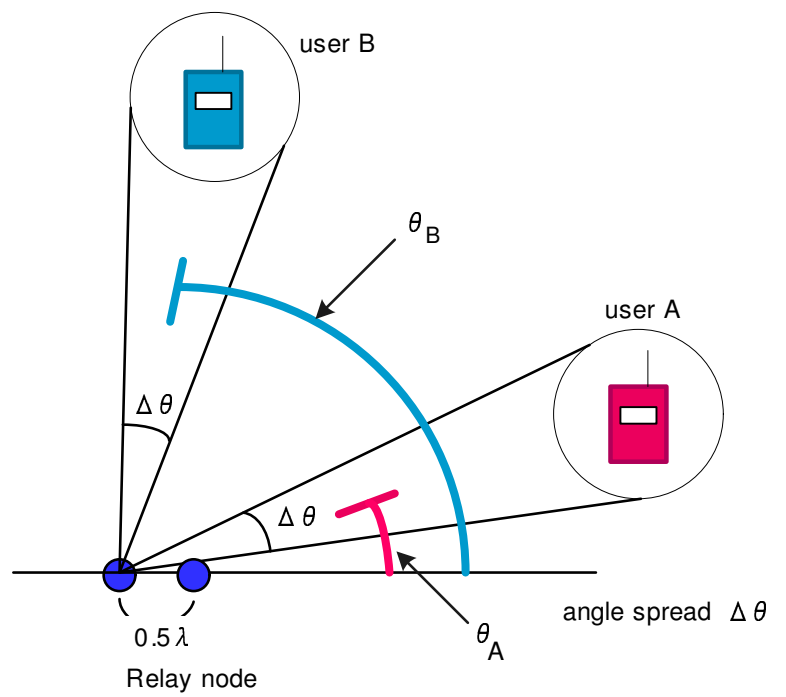

Fig. 2. Antenna array and user locations.

we assume that the SNR is high enough to approximate $1 \ll$ $P_{S} / P_{N}$. Then, (6) is rewritten as

$$
C \simeq \log \left(\left|\boldsymbol{h}_{a}^{T} \boldsymbol{w}\right|^{2}\left|\boldsymbol{h}_{b}^{T} \boldsymbol{w}\right|^{2}\right)+2 \log \left(\frac{P_{S}}{P_{N}}\right)
$$

Consequently, maximizing $C$ is simplified to maximizing $\left|\boldsymbol{h}_{a}^{T} \boldsymbol{w}\right|^{2}\left|\boldsymbol{h}_{b}^{T} \boldsymbol{w}\right|^{2}$.

Using the phase condition $a b^{*}=|a||b| e^{-j \phi}$, we have

$$
\left|\boldsymbol{h}_{a}^{T} \boldsymbol{w}\right|^{2}\left|\boldsymbol{h}_{b}^{T} \boldsymbol{w}\right|^{2}=A B(|a|+|b| \rho)^{2}(|b|+|a| \rho)^{2} .
$$

And $\boldsymbol{w}^{H} \boldsymbol{w}=1$ may be rewritten as

$$
\boldsymbol{w}^{H} \boldsymbol{w}=|a|^{2}+|b|^{2}+2|a||b| \rho=1 .
$$

Applying the method of Lagrange multipliers to (11) under the constraint (12) yields the very simple result:

$$
|a|=|b| \text {. }
$$

Finally, we obtain the weight vector

$$
\boldsymbol{w}_{\mathrm{c}}=\alpha\left(\frac{\boldsymbol{h}_{a}^{*}}{\sqrt{\boldsymbol{h}_{a}^{H} \boldsymbol{h}_{a}}}+\frac{\boldsymbol{h}_{a}^{H} \boldsymbol{h}_{b}}{\sqrt{\left|\boldsymbol{h}_{a}^{H} \boldsymbol{h}_{b}\right|^{2}}} \frac{\boldsymbol{h}_{b}^{*}}{\sqrt{\boldsymbol{h}_{b}^{H} \boldsymbol{h}_{b}}}\right),
$$

where $\alpha$ is a constant factor for $\boldsymbol{w}^{H} \boldsymbol{w}=1$.

Since (10) contains the product of both user's SNR, SNR imbalance occurs not so much unlike (3). Therefore, better performance than maximizing SNR is expected. Note that the condition (13) is approximately derived. Thus, the result (14) is not necessarily maximizing the total capacity especially when the SNR is low. The performance comparison between (14) and the optimum weight numerically-solved using (6) is briefly discussed in the Appendix.

\section{D. $S T B C$}

Alamouti's STBC is known as the robust way to obtain the transmit diversity gain [7]. Actually, it needs no beamforming. Here, however, we also consider the STBC for XOR-NC as in [8].
TABLE I

SIMULATION PARAMETERS.

\begin{tabular}{||c|c||}
\hline Number of transmit antenna & 2 \\
\hline Number of users & 2 \\
\hline Modulation & QPSK/16QAM/64QAM/256QAM \\
\hline Packet length & 128 symbols \\
\hline Channel coding & $\begin{array}{c}\text { Binary convolutional code } \\
\text { Code rate: 1/2, constraint length: 3 }\end{array}$ \\
\hline Channel model & $\begin{array}{c}\text { Quasi-static Rayleigh fading } \\
\text { (Jakes' Model) }\end{array}$ \\
\hline Antenna separation & $\lambda / 2$ \\
\hline Angle spread & $60^{\circ}$ \\
\hline Number of transmitted packets & 100,000 \\
\hline
\end{tabular}

\section{Systems Without XOR-NC}

In the system without XOR-NC, it is necessary to transmit a different signal to each user at the same time. Here, we consider the following two techniques categorized by an IUI suppression concept.

\section{A. SDMA}

SDMA is a technique to accommodate multiple users in a single channel. The transmitter (relay in this case) forms two different beam patterns which do not interfere with each other. Specifically, a null is directed to the other user's antenna element. Then, multiple unicasts are capable within the single time slot. SDMA loses the signal power efficiency since the power resource must be divided to multiple users. Moreover, SDMA consumes one degree of freedom for each single null. Thus, performance degradation is inevitable. In this paper, the transmit weight is determined using the MMSE algorithm. And, the transmit power is equally assigned to each user for the sake of simplicity, i.e, half of the XOR-NC case.

\section{B. $P H Y-N C$}

Considering that each user knows the waveform of its own packet, the IUI can be cancelled at the receiver using the channel information. The IUI cancellation mechanism is regarded as PHY-NC [2] [3]. If we assume perfect cancellation, the optimum transmit weight is defined as one maximizing each user's SNR. Then, the weight vectors for the users A and B are given by $\boldsymbol{h}_{a}^{*} / \sqrt{A}$ and $\boldsymbol{h}_{b}^{*} / \sqrt{B}$. Obviously, those are the same as the transmit maximal ratio combining (MRC). Thus, a better performance than SDMA is expected. In the paper, we assume that the transmit power is equally divided to each user as with SDMA.

\section{Numerical ANALYsis}

\section{A. Simulation Environment}

In this section, we show numerical results on the performance of systems with and without XOR-NC listed above. The simulation parameters are shown in Table I. For the sake of convenience, we assume that the relay node ideally receives all packets from each user without error and that the channel 


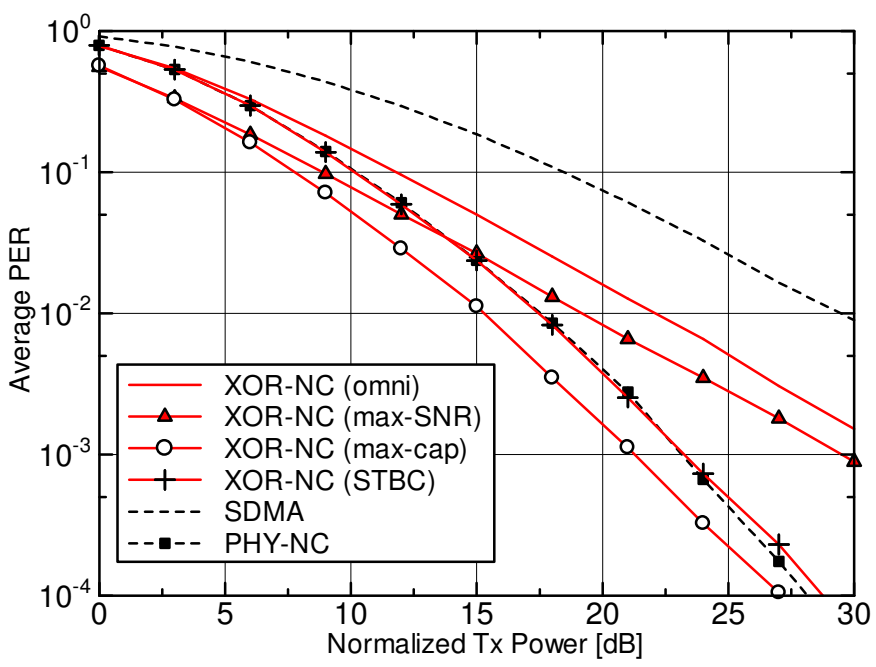

(a) Average PER

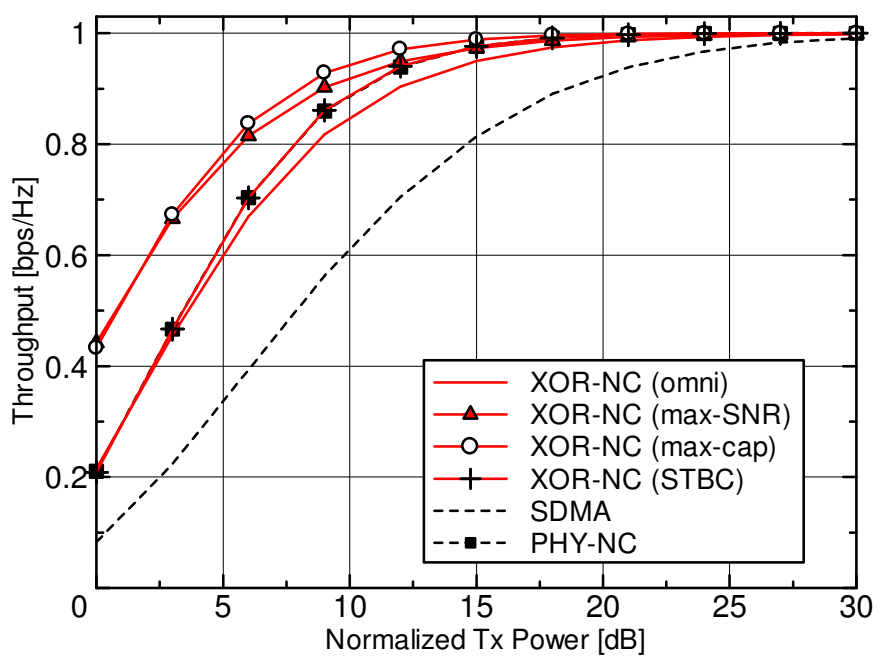

(b) Throughput

Fig. 3. PER and throughput performance for $\theta_{A}=10^{\circ}, \theta_{B}=40^{\circ}$.

information is perfectly known at the relay node and at both users. In SDMA and PHY-NC, the total transmit power is equalized to one in XOR-NC and equally distributed to both users. In the following, the number of antennas at the relay node is set to two since STBC is normally applied in the twoelement case. The antenna array and user locations are shown in Fig. 2. The angle spread is $60^{\circ}$ so that the diversity gain from beamforming is expected. The direction of user $\mathrm{A}$ is fixed to $\theta_{A}=10^{\circ}$, whereas the one of user $\mathrm{B}$ is alternated between $\theta_{B}=40^{\circ}$ and $100^{\circ}$. Except for the adaptive modulation case, the modulation is fixed to QPSK.

\section{B. Performance Comparison}

First, let us compare the performance for $\theta_{B}=40^{\circ}$. In this case, the angle difference between users is not large. Thus, the beamforming condition for XOR-NC is not so severe. In other words, some gain loss due to null-forming in SDMA would be observed.

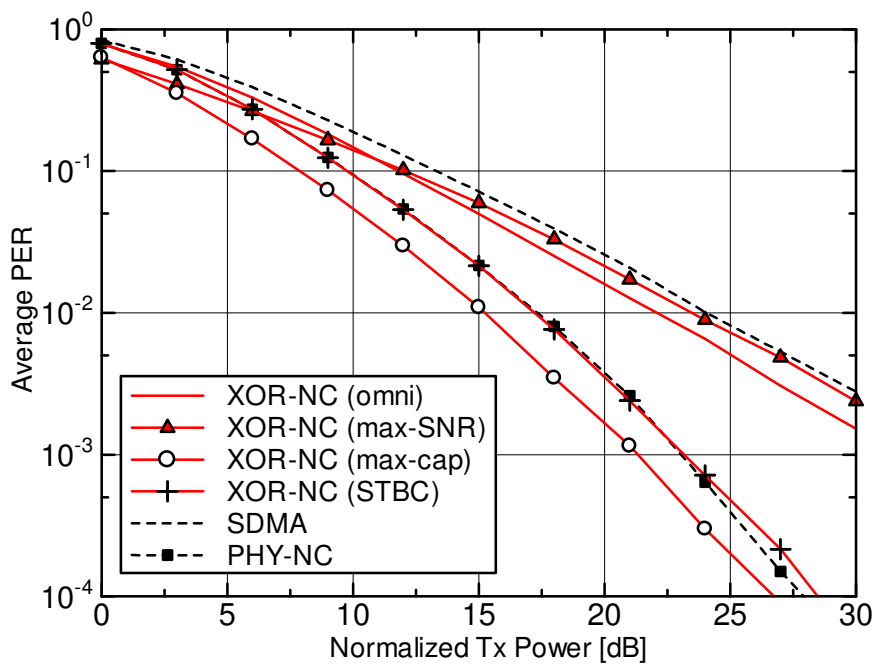

(a) Average PER

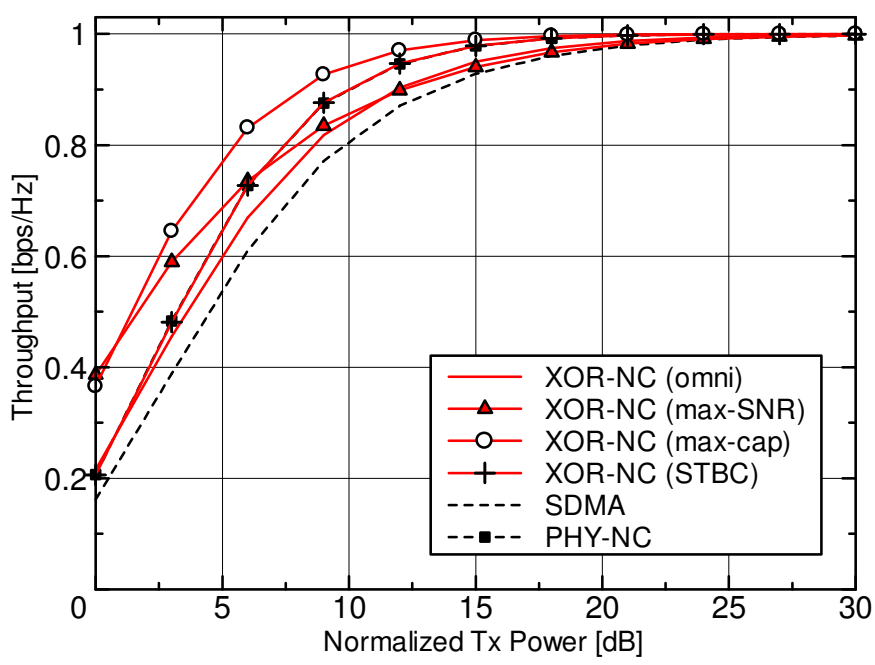

(b) Throughput

Fig. 4. PER and throughput performance for $\theta_{A}=10^{\circ}, \theta_{B}=100^{\circ}$.

Figure 3(a) shows the average packet error rate (PER) performance. It is clearly seen that the SDMA case is the worst. Since SDMA needs a degree of freedom for directing a null to another user, the diversity order is one. In addition, SDMA suffers from not only the transmit power loss but also the directing gain loss due to the null being closely-located to the desired user. Thus, about $6 \mathrm{~dB}$ degradation from the omni case is observed.

All curves for the XOR-NC cases are better than that of SDMA. In the omni and maximizing SNR (max-SNR) cases, however, the diversity order is one, as in the SDMA case. When observing the user-specific PER in the max-SNR case, the diversity order of the better SNR user is always two, whereas the worst user's diversity order is one. This seems to be caused by the max-SNR's property of emphasizing SNR imbalance. In other words, maximizing the sum of both users' SNR is almost the same as maximizing the SNR of the better 


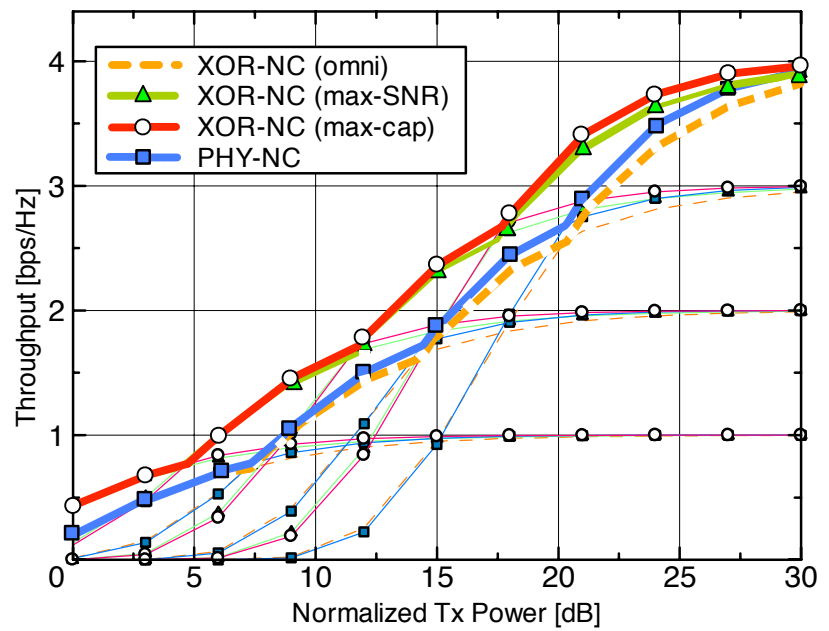

(a) $\theta_{A}=10^{\circ}, \theta_{B}=40^{\circ}$

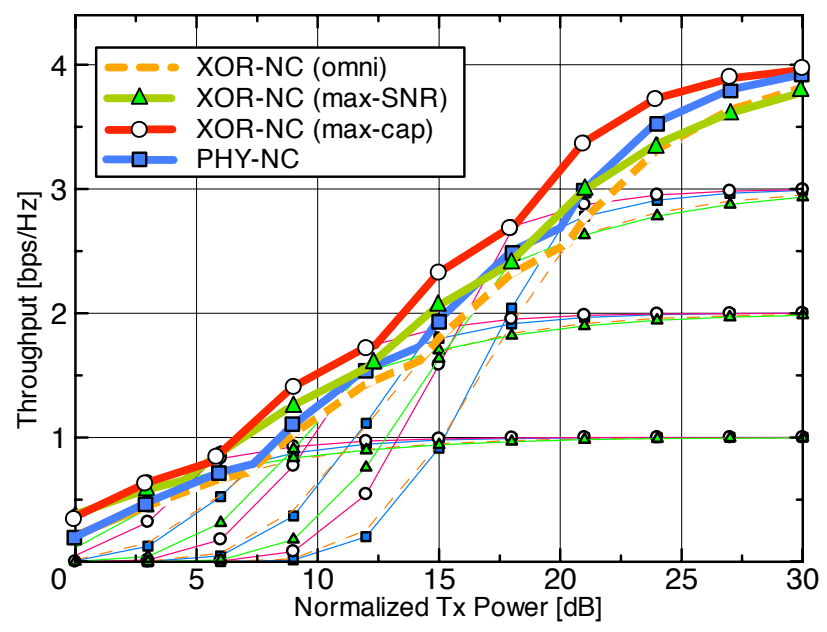

(b) $\theta_{A}=10^{\circ}, \theta_{B}=100^{\circ}$

Fig. 5. Throughput performance selecting the modulation from QPSK, 16QAM, 64QAM, and 256QAM.

user. Thus, no diversity effects are observed in the averaged PER performance.

The performance achieved through maximizing capacity (max-cap) is the best, and its diversity order is two. This result is very interesting since directing beams to both users consumes two degrees of freedom in general. However, the result implies that the max-cap strategy controls the beam pattern with proper balance. It can be seen that STBC also provides the diversity order of two. However, the transmit power loss due to assigning the power equally to both transmit antennas causes about $2-3 \mathrm{~dB}$ degradation from the max-cap case.

The diversity order of two is obtained also in the PHY-NC case since it utilizes the transmit MRC beam. However, the transmit power loss for sending two different packets degrades the performance compared to the one of max-cap. It should be noted that the performance is almost the same as the one of STBC since both methods have $3 \mathrm{~dB}$ power loss. Due to this power loss, max-SNR outperforms STBC and PHY-NC in the low SNR region.

Figure 3(b) shows the throughput performance. When PER $<0.01$, it is difficult to distinguish the performance difference in the throughput. Thus, the performance in the low SNR region becomes dominant in the figure. In fact, max-SNR provides the second best performance up to about $15 \mathrm{~dB}$, and the degradation at higher SNR is not significant. It can be said that max-SNR and max-cap are good choices as far as can be seen in the figure.

\section{Low Correlation Case}

Next, let us discuss the case when $\theta_{B}$ is $100^{\circ}\left(\theta_{A}=10^{\circ}\right)$, i.e., the users are separately located. In such a case, the gain loss of SDMA due to directing a null becomes smaller, whereas maintaining the gain at two different directions becomes difficult for XOR-NC. Thus, in comparison to the case of $\theta_{B}$ is $40^{\circ}$, the performance for the SDMA case is much improved as in Fig. 4 and almost reaching to that of the omni case. In contrast, the performance of max-SNR degrades severely. These facts indicate the orthogonality between spatial signatures of the users affects inversely to SDMA and maxSNR. However, even in the severer case for max-SNR as in Fig. 4, the performance is not worse than that of SDMA.

The curves of the other three cases for omni, STBC, and PHY-NC are equal to those in Fig. 3 since the performance is independent of another user's location. It should be noted that the performance of max-cap is almost the same as the one in Fig. 3 as well. This indicates that max-cap is a well-balanced strategy.

\section{Adaptive Modulation}

Finally, let us evaluate the performance for several modulation schemes. Fig. 5 shows the throughput performance when the adaptive modulation is utilized virtually. The thick curves correspond to those in the figures. In the case of $\theta_{A}=10^{\circ}$ and $\theta_{B}=40^{\circ}$, two curves for max-SNR and max-cap are almost overlapped. These two methods outperform the other two by about $2.5 \mathrm{~dB}$. However, only the performance of maxSNR degrades in the case of $\theta_{A}=10^{\circ}$ and $\theta_{B}=100^{\circ}$. It can be said that max-cap is the best criterion achieving both high performance and robustness with a small amount of complexity.

\section{CONCLUSION}

In this paper, we have investigated transmit beamforming techniques for XOR-NC when the relay node has two or more antenna elements and evaluated the packet error rate and throughput performance. The numerical analysis has indicated the effectiveness of the transmit beamforming in XORNC. Specifically, the criterion maximizing the sum capacity shows the best performance regardless of users' locations. Its optimum weight, solved in the paper, has a very simple form and is easy to calculate. Thus, we can conclude that the sum capacity criterion is very suitable for transmit beamforming for 


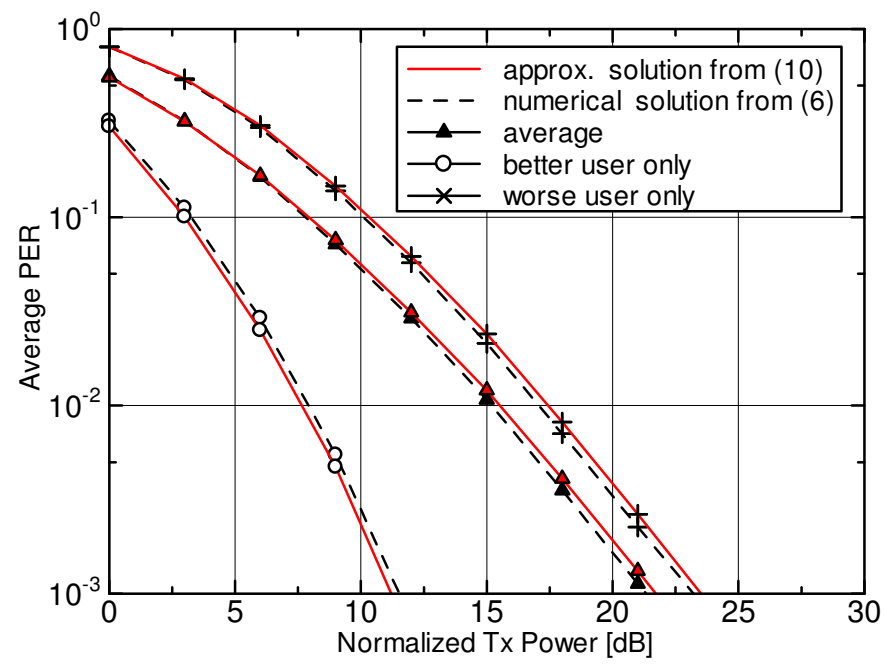

Fig. 6. PER performance for the maximizing capacity case with both approximated and optimum solutions.

multicast in XOR-NC. Application to multiuser MIMO systems and the evaluation of the impact of channel information error should be for further study.

\section{APPENDIX}

Let us discuss the accuracy of approximated solution (14). Unfortunately, the analytical solution maximizing (6) cannot be derived yet. Here, we have tried to solve it numerically. The amplitude of $a$ (and $b$ ) was changed from zero to one in small increments and the value maximizing (6) was taken. Fig. 6 shows the PER performance for the maximizing capacity case with both approximated and optimum (numerical) solutions. The other parameters are the same as the one in Fig. 3. In addition to the user-averaged PER, PERs for the better and worse users are also plotted.

The averaged PER performance with the optimum solutions is slightly degraded. This fact is surprising since the optimum solution actually yielded the maximum sum capacity ${ }^{1}$. The reason can be drawn by the other results shown in Fig. 6 . Clearly, the optimum solution has succeeded to improve the performance of the better user. However, the performance of the worse user deteriorates. This implies that the optimum solution emphasizes the SNR imbalance between users in order to maximize the sum capacity. Such imbalance emphasis never occurs in the approximated solution. Thus, we can conclude that the approximated solution is practically a good choice, although there might be other better criteria.

\section{ACKNOWLEDGMENT}

This work was supported by KAKENHI (21360175).

\footnotetext{
${ }^{1}$ The difference of the channel capacity of the approximated solution and the optimum solution is very small.
}

\section{REFERENCES}

[1] R. Ahlswede, N. Cai, S. Li, and R. Yeung, "Network information flow," IEEE Trans. Inform. Theory, vol. 46, pp. 1204-1216, July 2000.

[2] S. Zhang, S.-C. Liew, and P. P. Lam, "Physical-layer network coding," Proc. ACM MobiCom 2006, pp. 358-365, Sep. 2006.

[3] P. Popovski and H. Yomo, "Physical network coding in two-way wireless relay channels," Proc. IEEE ICC '07, pp. 707-712, June 2007.

[4] R. Zhang, Y.-C. Liang, C. C. Chai, and S. Cui, "Optimal beamforming for two-way multi-antenna relay channel with analogue network coding," IEEE J. Select. Areas Commun., vol. 27, no. 5, pp. 699-712, June 2009.

[5] N. Otuki, Y. Asai, T. Ichikawa, and M. Mizoguchi, "A packet combining demodulation scheme for multi-hop wireless systems using network coding," Proc. IEEE VTC 2008-Fall, Sep. 2008.

[6] S. C. Swales, M. A. Beach, D. J. Edwards, and J. P. McGeehan, "The performance enhancement of multibeam adaptive base-station antennas for cellular land mobile radio systems," IEEE Trans. Veh. Technol., vol. 39, no. 1, pp. 56-67, Feb. 1990.

[7] S. M. Alamouti, "A simple transmit diversity technique for wireless communication," IEEE J. Select. Areas Commun., vol. 16, no. 8, pp. 1451-1458 Oct. 1998.

[8] F. Ono and K. Sakaguchi, "Space time coded mimo network coding," Proc. PIMRC 2008, Sept. 2008 . 